\title{
MyHealthAvatar: personalized and empowerment health services through Internet of Things technologies
}

\author{
Emmanouil G. Spanakis \\ Institute of Computer Science \\ Foundation for Research and \\ Technology - Hellas \\ Heraklion, Crete, Greece \\ spanakis@ics.forth.gr \\ Dimitris Kafetzopoulos \\ Institute of Molecular Biology \\ and Biotechnology \\ Foundation for Research and \\ Technology - Hellas \\ Heraklion, Crete, Greece \\ kafetzo@imbb.forth.gr
}

\author{
Po Yang \\ Department of Computer \\ Science and Technology \\ University of Bedfordshire \\ Luton, Bedfordshire, UK \\ Po.Yang@beds.ac.uk
Kostas Marias
Institute of \\ Computer Science \\ Foundation for Research and \\ Technology - Hellas \\ Heraklion, Crete, Greece \\ kmarias@ics.forth.gr
}

\author{
Zhikun Deng \\ Department of Computer \\ Science and Technology \\ University of Bedfordshire \\ Luton, Bedfordshire, UK \\ Zhikun.Deng@beds.ac.uk \\ Manolis Tsiknakis \\ Department of Informatics \\ Engineering, \\ Technological Educational \\ Institute of Crete \\ Heraklion, Crete, Hellas \\ tsiknaki@ie.teicrete.gr
}

\author{
Vangelis Sakkalis \\ Institute of Computer Science \\ Foundation for Research and \\ Technology - Hellas \\ Heraklion, Crete, Greece \\ sakkalis@ics.forth.gr \\ Feng Dong \\ Department of \\ Computer Science and \\ Technology, \\ University of Bedfordshire \\ Luton, Bedfordshire, UK \\ Feng.Dong@beds.ac.uk
}

\begin{abstract}
The interconnection of heterogeneous data sources could provide a comprehensive picture of health parameters, thereby triggering an intervention by the medical staff upon detection of conditions that may lead to health deterioration, thus realizing preventive care. Supported Internet of Things technologies can be used to allow health related information to be locally aggregated and transmitted for remote monitoring and response. We present MyHealthAvatar (MHA), a personal digital health related collection bag, carried by individual citizens throughout their lifetime able to sustain in a meaningful manner all collected information. MHA acts as a unique companion continually following and empowering citizen and patients through a number of health related services. We describe the efforts on creating MHA patient-centered healthcare services for accessing, collecting and sharing long term multilevel personal health data through an integrated environment including: clinical data, genetic data, medical sensor data and devices, human behavior data and activity data for clinical data analysis, prediction and prevention for the individual citizen.
\end{abstract}

Keywords- internet of thins; digital patient; virtual physiological human; cloud computing; eHealth/mHealth;

\section{INTRODUCTION}

European healthcare systems have been subject to a long and complex history of independent evolution. As a result, the picture is highly fragmented with differences between member states, regions, and even between hospitals within the same country. So, from the perspective of the individual patient, maintaining a clinical record in a consistent manner is difficult, and the problem is being exacerbated by the increased population movement within Europe [1]. This situation poses as a threat to the provision of high quality healthcare services, and this is particularly true for the prediction and treatment of major, chronic and long-term diseases where a consistent record of individual patients is of great importance. To this end, information collection, access, sharing and analysis have become a key to the problem.

In the coming decades, the delivery model of healthcare is expected to transform from the present hospital-centric, to hospital-home-services [2] and finally to patient/citizen-centric services [3]. The rapid progress of computing power and ICT and Internet of Things technology [4] offers great potential for addressing challenges in information access, collection, sharing and analysis for new knowledge discovery, and has led to a huge amount of valuable data becoming available on the web. These newly available technologies grant us unprecedented opportunities to support next-generation healthcare in tackling, among other things, the ageing population and the impact of its growth on the numbers of patients suffering from chronic diseases. A major barrier today is how to inter-connect healthcare services, technology and people to enhance current offered healthcare services and applications [5].

Previous work has shown that Internet-of-Tings (IoT) can significantly contribute to the overall decrease of healthcare costs while increasing the health outcomes if it satisfies two conditions: enable the management of a particular disease and the mindset and behavioral changes of the stakeholders in the system [5], [6]. However, to take advantages of these technologies it is essential to study IoT systems in terms of performance, security, privacy, reliability and return-oninvestment. The work in [6] elaborates on the essential role of IoT in healthcare, their immense prospects and the usefulness of IoT in creating realistic health applications. -Internet of Things is a paradigm promising to manage citizens' digital 
identity for personalized healthcare services [8]. Wireless body area networks promise to revolutionize health monitoring, however, designers of such systems face a number of challenging tasks, as they need to address often quite conflicting requirements for connectivity [9], size, operating time, precision, and reliability [9].

In this work we focus on the state of the architectural requirements and considerations for the implementation as well as real life key internet of things technologies for the envisaged service platform. In this perspective IoT technologies can be used to enhance healthcare services and applications. We present MyHealthAvatar for the extension of the traditional health services into the physical realm of citizens, by means of the widespread deployment of spatially distributed devices and services. Below we describe the concept of MyHelathAvatar to argue that we can use Internet-of-Things technologies and the concept of the "digital patient" for future healthcare where digital and physical entities can be linked, by means of appropriate information and communication technologies, to enable a whole new class of applications and services.

\section{MYHEALTHAVATAR}

The VPH initiative has led to the collection and integration of predictive models and heterogeneous data to interpret and predict the progress of diseases and the effectiveness of treatments, which have laid down the foundation for new knowledge discovery. A number of VPH communities have started the movement to this direction by creating a digital patient road-map and by supporting data sharing infrastructures. Among, the most relevant projects is Discipulus ${ }^{1}$, which is a support action for producing a road-map for the future of digital patient, VPH Share ${ }^{2}$, p-Medicine ${ }^{3}, \mathrm{ACGT}^{4}$ and Tumor $^{5}$, of all which have shown a high level of interest in data sharing, owing to the importance of data in VPH research. The general concept of using digital avatars, a virtual digital bag of health related data, has been proposed by a number of systems. Typical examples include the 3D Avatar from $\mathrm{IBM}^{6}$, HealthVault from Microsoft ${ }^{7}$ and Google Health ${ }^{8}$. These systems have only shown limited impact on the current healthcare and after some time have stopped further development (i.e. like Google Health in 1st Jan, 20129). Also, similar work on the display of a human avatar was described in Zygote Body ${ }^{10}$, formally Google Body, which is a web application allows rendering and manipulation of 3D anatomical human bodies. However, it does not concern data collection for medical purposes.

MyHealthAvatar ${ }^{11}$ is a research initiative through which the feasibility of an innovative representation of the health status of citizens for future healthcare will be studied [11]. The goal is to create a digital avatar acting as a mediator between the endusers and health related data collections. It is envisaged as a personal container of heterogeneous sources of information

\footnotetext{
${ }^{1}$ http://www.digital-patient.net/

2 http://www.vph-share.eu/

3 http://www.p-medicine.eu/

4 www.eu-acgt.org

${ }^{5}$ http://tumor-project.eu/index.html

6 http://www-03.ibm.com/press/us/en/pressrelease/22375.wss
}

(medical, environmental, lifestyle) all blended in a single framework, utilizing modern information and communication technologies, providing long-term and consistent health information along a person's a timeline. The designed architectural platform (Figure 1) is an integrated facility that allows multiple functionalities rather than just a data storage facility as in the previous attempts. Its distinctive features include: Services to support data collection with minimal user input, including web information extraction, mobile apps, etc; A toolbox to support clinical decisions by using simulation models and by using visual analytics; Data and model repositories to provide rich resources of data and models; Ontology and RDF repositories to support data search and reasoning; A cloud based ICT architecture that allows the access of data from a range of different sources, and integration of the repositories, the toolbox and the ICT utilities.

\section{A. Architectural requirements}

The architectural blueprint and implementation for the definition and design of MyHealthAvatar is based on a number of components, standards and the technologies to meet specific user and functional requirements. We followed the IEEE 1471 recommendation 12 that defines an architecture as the fundamental organization of a system embodied in its components, their relationships to each other and to the environment and the principles guiding its design and evolution. The steps were on capturing stakeholder needs; making a series of architectural design decisions that resulted in a solution that meet these needs, assessing it against the stakeholder needs, and refining the solution.

MyHealthAvatar supports an infrastructure maximizing the yield of biomedical and health informatics research expenditure through integrated ICT capacities in terms of integrating the patient information into a coherent entity, which will subsequently offer medical professionals and researchers an interface for the access of a large set of patient information through the sharing of the avatar data, and for blending information with extreme heterogeneity, including those from different data sources, different models, organ systems, spacetime scales and modalities.

MyHealthAvatar uses innovative technologies to support data collection, search and reasoning, with minimal user effort. For example, mobile apps and social networks will be used to collect useful and valuable data from patients. Moreover, ontologies will support the annotation and transformation of multi-scale medical and social data and models in a unified way. This will facilitate data search and reasoning and the integration of data from a range of different resources (e.g. clinical, laboratory, social).

\footnotetext{
${ }^{7}$ https://www.healthvault.com/gr/en

${ }^{8}$ http://en.wikipedia.org/wiki/Google Health

${ }_{9}$ http://www.google.com/intl/en-US/health/faq.html

$10 \mathrm{http} / / /$ www.zygotebody.com/

${ }^{11} \mathrm{http}: / /$ www.myhealthavatar.eu/

12 http://cabibbo.dia.uniroma3.it/ids/altrui/ieee1471.pdf
} 


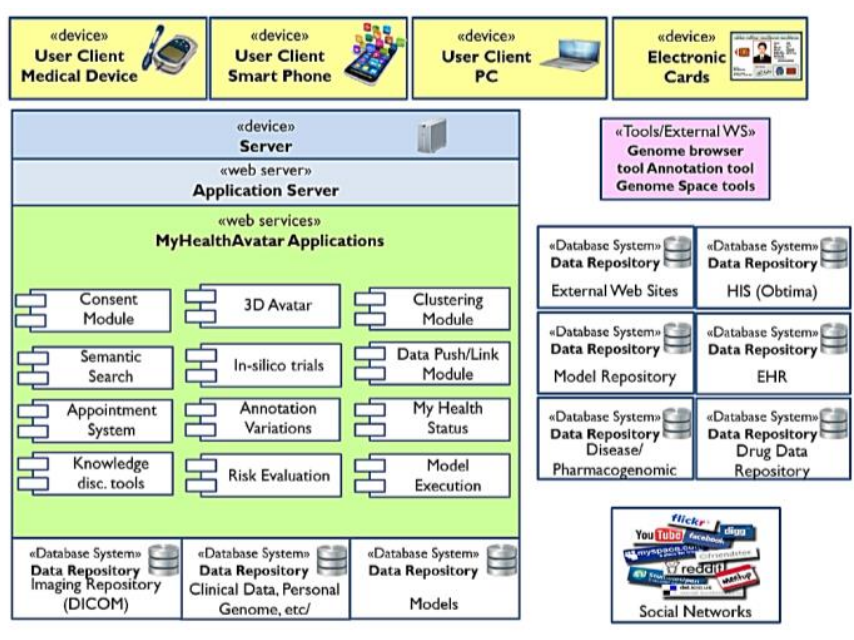

Figure 1. MyHealthAvatar Component Deployment

\section{B. MHA: As a Life Logging Data Aggregation Platform}

MyHealthAvatar uses information extraction through mobile applications to allow dynamic data collection along a timeline with minimal user effort. These data are useful to doctors in diagnosis and clinical decision making. Both smart phones and social media are supported by the latest technology and they have a huge number of participants and customers. It is expected that connecting the avatars to these facilities will significantly increase the level of participation from public.
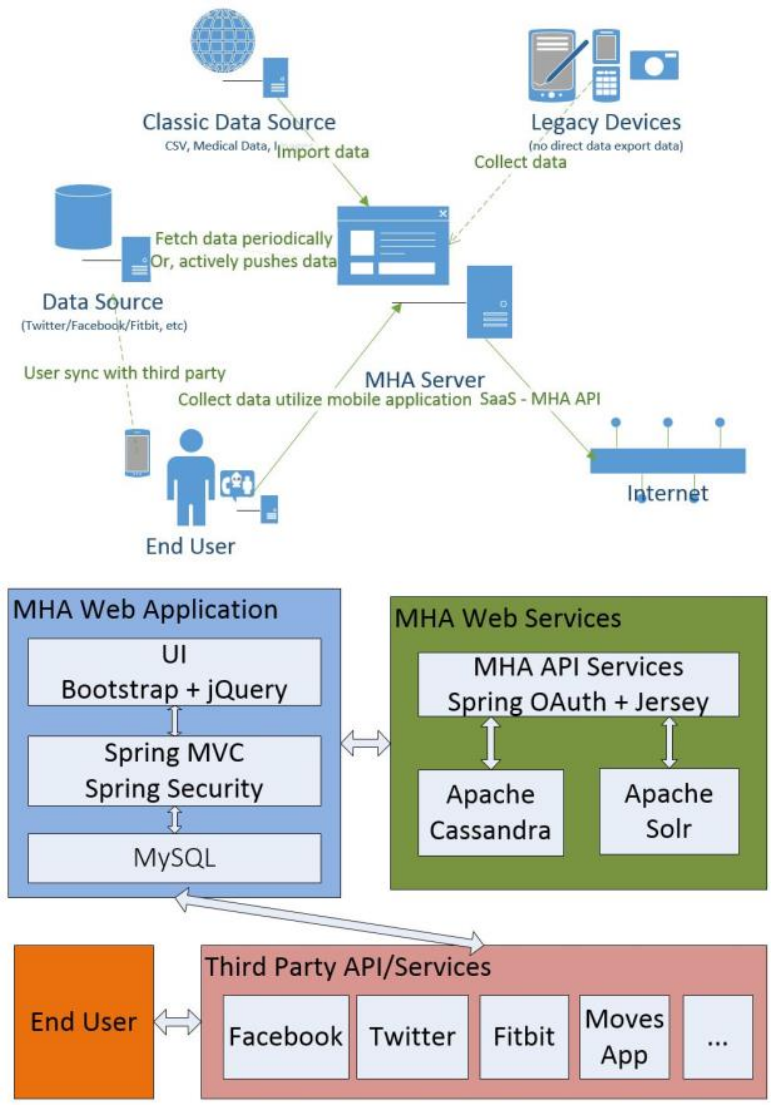

Figure 4. MyHealthAvatar Data Aggregator A Three-tier structure
In particular, mobile applications will monitor user's "health-status", "lifestyle" and "wellness" and upload data to the avatar system for close monitoring of health conditions and prevention of many diseases. Data collection and semantic interoperability is one of the keys to the success of the proposed avatar. Ontologies are used to identify and resolve heterogeneity problems, usually at schema level, as a means for establishing an explicit formal vocabulary to share. In order to design a life logging data collection aggregator, some essential requirements are identified as: a) Data Types related to physical activities and on health and physiological data; b) API support for iOS and Android systems. For most of mobile apps or wearable device, they actually support both iOS and Android system; c) Data protection and legal issue. So far there are no commercial applications or platforms practically implementing the extraction.

The implementation of a Life-logging data aggregator in MHA system (see figure 2) is to establish connections with providers and automatically retrieve data on behalf of MHA users. The key issues of implementation are:

SaaS: data collection from SaaS is a three-way conversation between a service providers (e.g. Fitbit server), service consumers (MHA platform) and a user who holds accounts on both provider and consumer. Some of the service providers have sophisticated functionalities which stores lots of different data of their users. All interaction between MHA and service providers are scoped to the context of user's health related data on the service provider side, and MHA will only retrieve the data which user specifically gives permission to.

OAuth $1 \&$ 2: OAuth is one of the most popular open stardanard authorization protocol which provides a mechanism for service consumer to access service provider's resources on behalf of the resource owner (e.g. the end-user). OAuth provide a process for end-users to authorize service consumer access to their data on service provider's server without sharing their credentials (username/password), in this platform is normally implemented using browser redirections.

Spring Social Framework: The main programming language choice is Java and Spring Framework powers the platform's web technology stack. The Spring Social Framework is the nature pick for doing OAuth communication. Combined with Spring Scurity Framework, the platform is able to deliver user a secure environment which is user-friendly and protects user's data.

Three-tier Structure: The presentation web UI , application service and data management functions are separated, which provide separation of concerns and create flexible and reusable web application. Different researchers can focus on one tier and implement independently and have its own release pace as you can see from following frontend and backend technology descriptions.

Backend: The implementation backend is based on Java programming language and Spring Framework technology stack, the frontend UI is mainly based on Twitter Bootstrap, jQuery and AngularJS. Java runs on all major platforms include Windows, Linux and Mac OS, which makes module based on Java is generally more portable between OSes, also JVM's 
proven high performance is crucial for our potential large user base. Spring Framework is the de-facto standard in enterprise Java programming, the developer team's high experience in Spring Framework mkes it our pick on implement the project.

Frontend: is a relatively separate Grunt project which uses Bower to manage the frontend JavaScript dependancies. The frontend soruce code is unit tested and built on a conituous integration manner. Twitter Bootstrap 3 is used to support both mobile and desktop browser, mobile first responsive design is applied for the web UI. AngularJS and jQuery JavaScript libraries are used to help shape the frontend logic, and interact with the REST service provided by backend. The combination of cutting-edge frontend technology stack does give us a enjoyable developing experience and a great dynamic userfriendly UI.

Server/Hosting: The project is hosted on Ubuntu 12.04 LTS VPS with all latest security pactches, Apache is used to server static content while Tomcat 7 is servlet container which provide dyamic contents (reverse proxied). For scalability and future growing larger user groups, a HAProxy server is configured to work as software load balancer and a Radis server is tested to be the cache server.

We performed a stability evaluation of the life-logging data aggregator by randomly retrieving these data every day in one month. The results are shown in Table.2. It appears that the lifelogging data aggregator can successfully gather the heterogeneous data from different resources with high success ratio. .Some challenging issues that may affect MHS platform performance are: a) third party APIs (mostly in beta version and under rapid development) may potentially constrain the performance of MHA platform as the number of MHA users growing; b) the unified data format may require a further definition as the type and volume of heterogeneous data increasing and c) legal and data protection issue need to be clearly concerned and clarified, particularly for social media resource.

Table 2: Stability evaluation of life-logging data aggregator

\begin{tabular}{l|ccc}
\hline Resource & $\begin{array}{c}\text { Times per } \\
\text { day }\end{array}$ & $\begin{array}{c}\text { Success } \\
\text { ratio }\end{array}$ & $\begin{array}{c}\text { Size per } \\
\text { request }\end{array}$ \\
\hline Fitbit & 20 & $95 \%$ & $850 \mathrm{~b}$ \\
MyTrack & 40 & $100 \%$ & $80 \mathrm{k}$ \\
Moves & 40 & $97.5 \%$ & $6.5 \mathrm{k}$ \\
Facebook & 40 & $97.5 \%$ & $67 \mathrm{k}$ \\
Twitter & 40 & $100 \%$ & $41 \mathrm{k}$ \\
\hline
\end{tabular}

\section{CONCLUSION}

The risks, developments and treatment of many major chronic diseases (neurological, cardiovascular, cancer, diabetes) are affected by a great number of individual health factors, genetic information, age, lifestyle and the environment. The dynamic and temporal nature of them could pose threats to the provision of high quality and integrated healthcare services. Therefore, gaining access to long-term and consistent data collection of these health factors could be particularly useful for supporting individualized prediction and treatment. Moreover, the collection of data across many individuals would lead to a huge and comprehensive population data resource, which would offer extremely valuable input to clinical research for new knowledge discovery. In many cases, there is a need to shift medical care from institutions to the home environment. To this end, ICT tools are being proposed and studied to reform the traditional ways in which medical data are recorded, tested and analyzed, without in any way reducing its quality. Digital avatar vision makes it possible to set up new interactions between doctors and patients, supporting individualized medicine and treatment. As an innovative concept, more effort is required to focus in addressing questions regarding user perspectives, use cases, scenarios, the power and limitations of modern technologies, the clinical acceptability of digital avatar in terms of supporting personalized medicine and potential legal and ethical aspects of digital avatars.

\section{ACKNOWLEDGMENTS}

This work has been supported by MyHealthAvatar EU project (http://www.myhealthavatar.eu/) funded by the European Commission under contract FP7-600929.

\section{REFERENCES}

[1] Migration Information Source, Available: http://www.migrationinformation.org/feature/display.cfm?ID=402

[2] Spanakis EG, Chiarugi F, Kouroubali A, Spat S, Beck P, Asanin S, Rosengren P, Gergely T, Thestrup J Diabetes Management Using Modern Information and Communication Technologies and New Care Models Interact J Med Res 2012;1(2):e8 doi: 10.2196/ijmr.2193 PMID: 23612026

[3] Koop, C.E.; Mosher, R.; Kun, L.; Geiling, J.; Grigg, E.; Long, S.; Macedonia, C.; Merrell, R.; Satava, R.; Rosen, J. (2008). Future delivery of health care: Cybercare. IEEE Engineering in Medicine and Biology Magazine, 27(6), 29-38.

[4] Daniele Miorandi, Sabrina Sicari, Francesco De Pellegrini, Imrich Chlamtac, Internet of things: Vision, applications and research challenges, Ad Hoc Networks, Volume 10, Issue 7, September 2012, Pages 14971516, ISSN 1570-8705, http://dx.doi.org/10.1016/j.adhoc.2012.02.016.

[5] Couturier, Jérôme and Sola, Davide and Borioli, Giovanni Scarso and Raiciu, Cristina, How Can the Internet of Things Help to Overcome Current Healthcare Challenges (2012). Communications \& Strategies, No. 87, 3rd Quarter 2012, pp. 67-81. SSRN: http://ssrn.com/abstract=2304133

[6] Spanakis, E.G., Lelis, P., Chiarugi, F., \& Chronaki, C. (2006). R\&D challenges in developing an ambient intelligence eHealth platform. EMBEC 2005, Prague, Czech Republic, November 2005., Prague, Czech Republic, 20-25 November (vol. 11, pp. 1727-1983).

[7] Mir Sajjad Hussain Talpur, The Appliance Pervasive of Internet of Things in Healthcare Systems, IJCSI Journal, Volume 10, Issue 1, No 1, January 2013, arXiv:1306.3953v1.

[8] Simonov, M., Zich, R., \& Flavia, M. (2008). Personalized Healthcare Communication in Internet of Things. Proc. of URSI GA08.

[9] Spanakis, E.G.; Sakkalis, V.; Marias, K.; Traganitis, A. Cross Layer Interference Management in Wireless Biomedical Networks. Entropy 2014, 16, 2085-2104.

[10] Chris Otto, Aleksandar Milenkovic, Corey Sanders and Emil Jovanov, System architecture of a wireless body area sensor network for ubiquitous health monitoring, Journal of Mobile Multimedia, Vol. 1, No.4 (2006) 307-326 Rinton Press.

[11] Maniadi, E.; Kondylakis, H.; Spanakis, E.G.; Spanakis, M.; Tsiknakis, M.; Marias, K.; Feng Dong, "Designing a digital patient avatar in the context of the MyHealthAvatar project initiative," Bioinformatics and Bioengineering (BIBE), 2013 IEEE 13th International Conference on , vol., no., pp.1,4, 10-13 Nov. 2013. 